\title{
On Polymer Conformations in Elongational Flows
}

\author{
T. Chan ${ }^{1}$, David S. Dean ${ }^{2}$, Kalvis M. Jansons ${ }^{3}$, L.C.G. Rogers ${ }^{4}$ \\ ${ }^{1}$ Dept of Actuarial Maths. \& Statistics, Heriot-Watt University, Riccarton, Edinburgh \\ EH14 4AS, U K. \\ ${ }^{2}$ Dept. of Applied Maths \& Theo. Phys, University of Cambridge, Silver Street, Cambridge \\ CB3 9EW, U.K. \\ 3 Dept. of Mathematics, University College, Gower Street, London WC1E 6BT, U.K. \\ ${ }^{4}$ School of Math Sciences, Queen Mary \& Westfield College, Mile End Road, London E1 4NS, \\ $\mathrm{UK}$
}

Received: 9 March 1992/in revised form 18 June 1993

\begin{abstract}
We consider various models of polymer conformations using paths of Gaussian processes such as Brownian motion. In each case, the calculation of the law of the moment of inertia of a random polymer structure (which is equivalent to the calculation of the partition function) is reduced to the problem of finding the law of a certain quadratic functional of a Gaussian process. We present a new method for computing the Laplace transforms of these quadratic functionals which exploit their special form via the Ray-Knight Theorem and which does not involve the classical method of eigenvalue expansions. We apply the method to several simple examples, then show how the same techniques can be applied to more complicated cases with the aid of a little excursion theory.
\end{abstract}

\section{Introduction}

This study extends and greatly simplifies previous work on polymer conformations in pure straining motions (or elongational flows). The main method used is a technique for characterizing the law of a quadratic functional of Brownian motion (or other Gaussian process) which also extends and greatly simplifies previous work in this area. Among other examples we consider the conformations of single-chain polymers with one end attached to a suspended particle and ring polymers, in an elongational flow at zero Reynolds number, that is, in a limit where inertia forces are neglected. In each case we compute the law of the moment of inertia of the random polymer. The laws of other functionals can also be computed, but we concentrate on the moment of inertia as a particularly important physical quantity. To understand why we can convert the polymer problem into one on quadratic functionals of Gaussian processes, we first present some of the physics of the problem.

An elongational (or straining) velocity field $u(x)$ is described by

$$
u(x)=E x
$$

where $x$ is the position vector and $E$ is a traceless symmetric matrix called the rate-of-strain tensor. The force on a single rigid particle in a zero Reynolds number 
flow is given by

$$
F=R u,
$$

where $R$ is the resistance tensor of the particle; for simplicity we assume that $R$ is isotropic, so that $R$ may be regarded as the scalar resistance of the particle in (1.2). For the isotropic case, the work done against an elongational flow is conservative, with the potential energy $V(x)$ of a particle moved from 0 to $x$ given by

$$
V(x)=-\frac{1}{2} R x^{T} E x
$$

If we consider a single-chain polymer as a Brownian path threaded through infinitesimal isotropic particles, and neglect the effects of hydrodynamic interactions between the infinitesimal particles and self avoidance, we can define the flow energy of the polymer by

$$
V(X)=-\frac{1}{2} \int_{0}^{\tau} X_{t}^{T} E X_{t} R(d t)
$$

where $X_{t}, 0 \leqq t \leqq \tau$ is a three-dimensional Brownian motion describing the polymer path before Boltzmann re-weighting and $R$ is the resistance measure along the polymer. Without loss of generality we may take $\tau=1$ and $R[0,1]=1$. The functional $V$ is not quite appopriate for considering the statistical mechanics of polymer shapes in an elongational flow, since it is not invariant under translations of the whole polymer. To achieve translational invariance we must change to centre-of-resistance coordinates, namely redefine the flow energy of the polymer by

$$
V(X)=-\frac{1}{2} \int_{0}^{1}\left(X_{t}-\bar{X}\right)^{T} E\left(X_{t}-\bar{X}\right) R(d t),
$$

where $\bar{X}=\int_{0}^{1} X_{t} R(d t)$ is the centre of resistance. The statistical mechanical ensemble average of any functional $F$ of the polymer shape is given by

$$
Z^{-1} \mathbb{E}\left\{F(X) e^{-V(X) / k T}\right\},
$$

where $Z=\mathbb{E}\left\{e^{-V(X) / k T}\right\}$ is the partition function and $k T$ is the temperature in energy units, which we set equal to unity. Notice that the partition function factorizes:

$$
Z=\prod_{i=1}^{3} Z_{i}
$$

where

$$
Z_{i}=\mathbb{E}\left[\exp \left\{\frac{\lambda_{i}}{2} \int_{0}^{1}\left(X_{t}^{i}-\bar{X}^{i}\right)^{2} R(d t)\right\}\right],
$$

$\lambda_{i}$ being the eigenvalues of $E$ and $X^{i}$ the corresponding components of $X$. Thus in calculations where the functional $F$ also factorizes, the three-dimensional system reduces to three independent one-dimensional systems and the calculation of the partition function is reduced to the problem of calculating the law of the quadratic functional (1.8) of Brownian motion. In the sequel we restrict attention to onedimensional systems and drop the index $i$.

The result on quadratic functionals which we shall make use of is the following. 
Theorem 1.1. Let $\left(X_{t}\right)_{0 \leqq t \leqq 1}$ be either a Brownian motion started at 0 or a Brownian bridge, $X_{0}=X_{1}=0$, and let $m_{1}$ and $m_{2}$ be two finite measures on $[0,1]$. Then

$$
\begin{aligned}
& \mathbb{E}\left[\exp \left\{-\lambda \int_{0}^{1} X_{s}^{2} m_{1}(d s)-2 \lambda \int_{0}^{1} X_{s} m_{2}(d s)\right\}\right] \\
& =\mathbb{E}\left[\exp \left\{-\lambda \int_{0}^{1} X_{s}^{2} m_{1}(d s)\right\}\right] \exp \left\{\lambda^{2} \int_{0}^{1} \int_{0}^{1} \tilde{r}_{\lambda}^{\partial}(s, t) m_{2}(d t) m_{2}(d s)\right\},
\end{aligned}
$$

where $\tilde{r}_{\lambda}^{\partial}(\cdot, \cdot)$ is the resolvent density (with respect to $m_{1}$ ) of the diffusion in natural scale in $[0,1]$ with speed measure $m_{1}$, killed at 0 and reflected at 1 in the case where $X$ is Brownian motion, and killed at both 0 and 1 when $X$ is Brownian bridge.

This theorem is itself a special case of a more general result about Markov processes, which we state here for finite Markov chains.

Theorem 1.2. Let $A$ be the generator of an irreducible transient Markov chain on a finite state space $I$, which is symmetrizable with respect to the diagonal matrix $D$ :

$$
D A=A^{T} D \text {. }
$$

Let $\xi \equiv\left(\xi_{i}\right)_{i \in I}$ be the zero-mean Gaussian vector with covariance matrix

$$
V=\frac{1}{2} G D^{-1}=-\frac{1}{2} A^{-1} D^{-1}
$$

where $G=-A^{-1}$ is the Green function of the Markov process. Suppose $\Gamma$ is a diagonal matrix with strictly positive diagonal entries. Then

$$
\begin{array}{r}
\mathbb{E}\left[\exp \left\{-\lambda(\xi+a)^{T} \Gamma D(\xi+a)+\lambda a^{T} \Gamma D a\right\}\right] \\
\left.=\mathbb{E}\left[\exp \lambda \xi^{T} \Gamma D \xi\right\}\right] \exp \left\{\lambda^{2} a^{T} \Gamma D \tilde{R}_{\lambda} a\right\},
\end{array}
$$

for any vector a. Here $\tilde{R}_{\lambda}=\left(\lambda-\Gamma^{-1} A\right)^{-1}$ is the resolvent of the chain time-changed by $\Gamma$.

The proof of these two results occupies Sect. 2. In Sect. 3 we apply this, together with ideas from excursion theory to compute:

(i) the joint law of $\left(\int_{0}^{1} B_{s}^{2} d s, \int_{0}^{1} B_{s} d s\right)$, where $B$ is Brownian motion;

(ii) the joint law of $\left(\int_{0}^{1} B_{s}^{2} \mu(d s), \int_{0}^{1} B_{s} \mu(d s)\right)$, where

$$
\mu(d s)=\left(\alpha^{2} 1_{[0, a]}(s)+\beta^{2} 1_{[a, 1]}(s)\right) d s ;
$$

(iii) the joint law of $\left(\int_{0}^{1} X_{s}^{2} d s, \int_{0}^{1} X_{s} d s\right)$, where $X$ is Brownian bridge;

(iv) the joint law of $\left(\int_{\mathscr{T}} X_{s}^{2} d s, \int_{\mathscr{T}} X_{s} d s\right)$, where $X$ is a star polymer indexed by a tree $\mathscr{T}$.

In each case, a trivial technique yields immediately the moment of inertia. We then proceed at a more general level to obtain expressions for:

(v) the joint law of $\left(\int_{\mathscr{T}} X_{s}^{2} \mu(d s), \int_{\mathscr{T}} X_{s} \mu(d s)\right)$, where $X$ is a Brownian motion indexed by a general tree $\mathscr{T}$;

(vi) as for (v) but with $X$ a ring polymer with attached trees;

(vii) as for (ii) but with $\mu(d s)=\alpha^{2} / 2 d s+k_{0} \delta_{0}(d s)+k_{1} \delta_{1}(d s)$.

Finally, we describe how to build up more complicated polymer shapes. 


\section{Probabilistic Treatment of Quadratic Functionals of Brownian Motion}

It is clear that if we can evaluate a Laplace transform of the form

$$
\mathbb{E}\left[\exp \left\{-\frac{\theta^{2}}{2} \int_{0}^{1}\left(X_{s}-a(s)\right)^{2} m(d s)\right\}\right],
$$

where $a$ is some deterministic function and $m$ is some finite measure on $[0,1]$, then we can evaluate the factors (1.8) of the partition function by taking $a$ to be a constant.

Laplace transforms of quadratic functionals of the form (2.1) can of course be calculated by the classical method of eigenfunction expansions; the papers of Lévy (1951), Fixman (1962), Krée (1986), McAonghusa and Pulé (1989), Chiang, Chow and Lee (1991), Chan (1991), Helfer and Zhao (1992) all discuss aspects of this method. The laws of various quadratic functionals are discussed in these papers, and also in Duplantier (1989), Yor (1989), Gaveau (1977), Donati-Martin and Yor (1991). The last reference makes effective use of a different technique, namely the stochastic Fubini theorem, to transform one problem into a seemingly unrelated one which may be easier to solve. The purpose of this section, however, is to show how expectations such as (2.1) can be computed by exploiting the key fact that the covariance of the Gaussian process is the Green function of a symmetrizable Markov process. This structural feature is widely used in the literature of random fields see, for example, Dynkin $(1980,1981)$, Williams (1973). This observation on its own is not sufficient to give the explicit formulae we seek, and the concrete nature of the Brownian motion (or Brownian bridge) enters through the Ray-Knight theorem on Brownian local time to give the final answers. The idea that Ray-Knight type computations involving integrals of local times are in some sense equivalent to computations involving quadratic functionals has been used before, for example in McGill's direct proof of the Ray-Knight Theorem (McGill (1981)).

There is at least one other different technique for such problems, which are shown to be equivalent to certain well-studied problems of optimal control by Rogers and Shi (1992).

First, let us recall the following important result which features prominently in statistics:

Lemma 2.1. Let $X$ be an $\mathbb{R}^{d}$-valued random variable with $N(0, V)$ distribution for some covariance matrix $V$ and let $a \in \mathbb{R}^{d}$. Then if $Q$ is a positive-definite symmetric matrix, the following identity holds:

$$
\begin{aligned}
\mathbb{E}[\exp \{ & \left.\left.-\frac{1}{2}(X+a)^{T} Q(X+a)\right\}\right] \\
& =\operatorname{det}(I+Q V)^{-1 / 2} \exp \left\{-\frac{1}{2} a^{T}(I+Q V)^{-1} Q a\right\} .
\end{aligned}
$$

Proof. Express the left-hand side of (2.2) as an integral over $\mathbb{R}^{d}$ with respect to the Gaussian density, and complete the square inside the exponential.

Lemma 2.1 is sometimes called the "fundamental theorem of statistics," since all results concerning the joint distributions of sums of squares of Gaussian variables can be deduced from it. 
The result can be generalized to infinite dimensions, but we keep to the finite-dimensional setting and deduce the results we want by soft weak convergence arguments, rather than get involved in the technicalities of unbounded operators.

Firstly, note that by taking

$$
V=-\frac{1}{2} A^{-1} D^{-1}, \quad Q=2 \lambda \Gamma D
$$

a little elementary matrix algebra yields Theorem 1.2 quickly from Lemma 2.1. The fact that $\Gamma D=D \Gamma$ is used in an essential way. Next, we use Theorem 1.2 to prove Theorem 1.1.

Proof of Theorem 1.1. We treat only the case where $X$ is Brownian motion, the other case being similar.

Let $\mathbb{D}_{n}=\left\{j 2^{-n}: j=1,2, \ldots, 2^{n}\right\}$ and firstly suppose that $m_{1}$ is concentrated on $\mathbb{D}_{n}, m_{2}$ is concentrated on $\mathbb{D}_{k}, k \leqq n$, with

$$
\frac{d m_{2}}{d m_{1}}(t)=a(t) \text {. }
$$

The left-hand side of (1.9) is of exactly the same form as the left-hand side of (1.12), since the integrals are only sums. To be explicit, the matrices are

$$
D=\operatorname{diag}\left(2^{-n}\right), \quad \Gamma=\operatorname{diag}\left(2^{n} m_{1}\left(\left\{j 2^{-n}\right\}\right)\right), \quad A_{j, j+1}=A_{j, j-1}=2^{n-1} .
$$

Theorem 1.2 is immediately applicable and yields Theorem 1.1 for this case.

Next, we generalize to arbitrary $m_{1}$, while keeping fixed the measure $m_{2}$ concentrated on $\mathbb{D}_{k}$. Of course, we pick $m_{1}^{(n)}$ concentrated on $\mathbb{D}_{n}$ such that $m_{1}^{(n)} \Rightarrow m_{1}$. The only piece of the weak convergence argument needing care is the convergence of the resolvent densities on the right-hand side of (1.9). But this is evident from the expression (50.10) in $\S \mathrm{V} .50$ of Rogers and Williams (1987):

$$
\tilde{r}_{\lambda}^{\partial}(x, y)=\mathbb{E}^{x} \int_{0}^{\infty} \exp \left\{-\lambda A_{u}\right\} l^{y}(d u),
$$

where $\left\{l_{t}^{y}: y \geqq 0, t \geqq 0\right\}$ is the local time process of a Brownian motion $W$ stopped at $\tau:=\inf \left\{t: W_{t}=0\right\}$ and

$$
A_{t}= \begin{cases}\int m_{1}(d y) l_{t}^{y} & t<\tau \\ +\infty & t \geqq \tau .\end{cases}
$$

The final extension to arbitrary $m_{2}$ is now immediate.

While (1.9) may not at first sight seem very explicit, as it involves the $\lambda$-resolvent density, the examples in the next section will show that in many cases with important applications explicit formulae for $\tilde{r}_{\lambda}^{\partial}$ can be found and the laws of most of the quadratic functionals which are of interest to us can be obtained this way.

Theorem 1.1 permits us to pass easily from the case $m_{2} \equiv 0$ to general $m_{2}$, but gives no useful information about the case $m_{2} \equiv 0$. However, in this case the right-hand side of (1.9) can be calculated very easily if $X$ is a Brownian motion or a Brownian bridge by means of a simple application of the famous Ray-Knight theorem, (part of) which states the following:

Theorem 2.2 (Ray, Knight). (i) Let $W_{t}$ be a Brownian motion with values in $(0,1]$, started at 1 and reflected at 1 , let $l_{t}^{a}, a \leqq 1$ be (the continuous version of) the 
(semi-martingale) local time for $W$ at $a$ and let $\tau=\inf \left\{t: W_{t}=0\right\}$. For independent Brownian motions $B_{1}(t), B_{2}(t)$ starting from 0 , let $Z_{t}=B_{1}(t)^{2}+B_{2}(t)^{2}$ be a squared two-dimensional Bessel process. Then the two processes $\left\{l_{\tau}^{a}: 0 \leqq a \leqq 1\right\}$ and $\left\{Z_{a}: 0 \leqq a \leqq 1\right\}$ are identical in law.

(ii) Let $R_{t}$ be a three-dimensional Bessel process with $R_{0}=0$, let $L_{t}^{a}$ be its local time process and let $T=\inf \left\{u: R_{u}=1\right\}$. Let $Y_{t}$ be a two-dimensional squared Bessel bridge. Then the processes $\left\{L_{T}^{a}: 0 \leqq a \leqq 1\right\}$ and $\left\{Y_{a}: 0 \leqq a \leqq 1\right\}$ are identical in law.

Proof. Part (i) is proved in Rogers and Williams (1987, §VI.52). Part (ii) can be proved using the famous path decomposition results of Williams; it follows directly by combining the path decomposition result of Theorem 2 in Williams (1970) and Theorem 4 in Williams (1970). (Theorem 4 of Williams (1970) is in turn a direct result of (i) above, together with Theorems 1 and 2 of Williams (1970), the last two of which are proved in Williams (1974).)

Putting

$$
\phi(\theta)=\mathbb{E}^{0}\left[\exp \left\{-\frac{\theta^{2}}{2} \int_{0}^{1} B_{s}^{2} m_{1}(d s)\right\}\right],
$$

where $B$ is now a Brownian motion, a direct application of the Ray-Knight theorem gives in particular

$$
\phi^{2}=\mathbb{E}^{1}\left[\exp \left\{-\frac{\theta^{2}}{2} \int_{0}^{1} l_{\tau}^{s} m_{1}(d s)\right\}\right] .
$$

Consider now the right-hand side of (2.3). Define

$$
\gamma_{t}:=\inf \left\{u: \int_{0}^{1} l_{u}^{s}(d s)>t\right\} .
$$

Let $\tilde{W}$ be a time-change of a Brownian motion $W$ on $(0,1]$ started at 1 and reflected at 1: $\tilde{W}_{t}=W\left(\gamma_{t}\right)$; moreover the local time processes $\tilde{l}_{t}^{a}$ for $\tilde{W}$ is also a time-change of the local time $l_{t}^{a}$ for $W$ and $\tilde{l}_{t}^{a}=l^{a}\left(\gamma_{t}\right)$ (see Rogers and Williams $(1987, \S \mathrm{V} .49)$ ). The local time $\widetilde{l}_{t}^{a}$ also serves as an occupation density for $\tilde{W}$ in the sense that for any bounded measurable function $f$

$$
\int_{0}^{t} f\left(\tilde{W}_{s}\right) d s=\int_{0}^{1} f(a) \tilde{l}_{t}^{a} m_{1}(d a) .
$$

Now let $H_{0}=\inf \left\{u: \tilde{W}_{u}=0\right\}$ and let $\tau$ be as in Theorem 2.2. Then we see that $H_{0}=\inf \left\{u: \gamma_{u}>\tau\right\}$ and in particular $\gamma\left(H_{0}\right)=\tau$. Putting all these together, we have

$$
\begin{aligned}
\int_{0}^{1} l_{\tau}^{s} m_{1}(d s) & =\int_{0}^{1} l_{\gamma\left(H_{0}\right)}^{s} m_{1}(d s) \\
& =\int_{0}^{1} \tilde{l}_{H_{0}}^{s} m_{1}(d s)=\int_{0}^{H_{0}} d t=H_{0} .
\end{aligned}
$$

Putting (2.3) and (2.4) together then gives

$$
\phi^{2}=\operatorname{det}\left(I+\theta^{2} Q V\right)^{-1}=\mathbb{E}^{1}\left[e^{-\theta^{2} H_{0} / 2}\right] .
$$

If $m_{1}$ is Lebesgue measure on $[0,1]$, we have $\gamma_{t}=t$ and it is well-known that $\phi^{2}=(\cosh \theta)^{-1}$ is the Laplace transform of the hitting time of 0 for $W$ (see, for example, Proposition II.3.7 of Revuz and Yor (1991)). 
Let $\left\{X_{t}: t \in[0,1]\right)$ now be a Brownian bridge from 0 to 0 . We need only consider the case of a Brownian bridge $X$ from 0 to 0 , since a bridge $\tilde{X}$ from 0 to $c$ can be got by adding a deterministic drift $c t$ to $X: \tilde{X}_{t}=X_{t}+c t$ and this will only contribute a deterministic term to the quadratic functional in (2.1) which can then be absorbed into the function $a(\cdot)$. As before, putting

$$
\phi(\theta)=\mathbb{E}^{0,0}\left[\exp \left\{-\frac{\theta^{2}}{2} \int_{0}^{1} X_{s}^{2} m_{1}(d s)\right\}\right],
$$

we have

$$
\phi^{2}=\mathbb{E}^{0}\left[\exp \left\{-\frac{\theta^{2}}{2} \int_{0}^{1} L_{T}^{s} m_{1}(d s)\right\}\right],
$$

where $L$ is the local time of a three-dimensional Bessel process $R$ starting from 0 as given in the Ray-Knight theorem. Time-changing the three-dimensional Bessel process $R$ via

$$
\Gamma_{t}:=\inf \left\{u: \int_{0}^{1} L_{u}^{s} m_{1}(d s)>t\right\}
$$

and using the same treatment as before shows that

$$
\phi^{2}=\operatorname{det}\left(I+\theta^{2} Q V\right)^{-1}=\mathbb{E}^{0}\left[e^{-\theta^{2} H_{1} / 2}\right],
$$

where $H_{1}$ is the hitting time of 1 for the process $R\left(\Gamma_{t}\right)$. If $m_{1}$ is Lebesgue measure, an application of the Optional Stopping Theorem to the martingale $e^{-\theta^{2} t / 2} R_{t}^{-1} \sinh \left(\theta R_{t}\right)$ shows that $\phi^{2}=\theta / \sinh \theta$.

In most of the applications later, the function $a$ in (2.1) is a constant: $a \equiv x$ for some $x \in \mathbb{R}$. In this case, the identity (1.9) is easily worked to the form

$$
\begin{aligned}
& \mathbb{E}\left[\exp \left\{-\lambda \int_{0}^{1}\left(X_{s}+x\right)^{2} m_{1}(d s)\right\}\right] \\
& \quad=\mathbb{E}\left[\exp \left\{-\lambda \int_{0}^{1} X_{s}^{2} m_{1}(d s)\right\}\right] \exp \left\{-\lambda x^{2} \int_{0}^{1}\left(1-\lambda\left(\tilde{R}_{\lambda}^{\partial} 1\right)(s)\right) m_{1}(d s)\right\}
\end{aligned}
$$

(where $\tilde{R}_{\lambda}^{\partial}$ is the operator associated with the resolvent density $\tilde{r}_{\lambda}^{\partial}(\cdot, \cdot)$ ), which has an interpretation in terms of results from excursion theory. In fact, we have already seen the relevance of excursion theory to this problem in the earlier applications of the Ray-Knight theorem, which is proved by excursion arguments that can be modified to give similar results for other processes. (The survey article Rogers (1989) contains an introduction to the basic ideas and results in excursion theory, as well as good references to the literature where the interested reader can pursue the details.) Consider first the case that $X$ in (2.7) is a Brownian motion. Let $\tilde{W}^{\partial}$ be the killed process whose resolvent operator is $\tilde{R}_{\lambda}^{\partial}, H_{0}=\inf \left\{t: \tilde{W}_{t}^{\partial}=0\right\}$ and define

$$
\psi_{\lambda}(y)=\mathbb{E}^{y}\left[e^{-\lambda H_{0}}\right] \text {. }
$$

Then $\psi_{\lambda}$ may be expressed as

$$
\psi_{\lambda}(y)=1-\left(\lambda \tilde{R}_{\lambda}^{\partial} 1\right)(y) .
$$

It is well-known from excursion theory (see, for example, Rogers (1983)) that a process $\tilde{W}$ can be obtained by extending the killed process $\tilde{W}^{\partial}$ beyond the time of 
death $H_{0}$; this may be done by defining the resolvent $\tilde{R}_{\lambda}$ of $\tilde{W}$ via the identity

$$
\tilde{R}_{\lambda} f(x)=\tilde{R}_{\lambda}^{\partial} f(x)+\psi_{\lambda}(x) \tilde{R}_{\lambda} f(0) .
$$

Thus in order to obtain $\tilde{R}_{\lambda} f$ from $\tilde{R}_{\lambda}^{\partial} f$, we need only calculate $\tilde{R}_{\lambda} f(0)$. We shall see in a moment how to obtain $\tilde{R}_{\lambda} f(0)$.

Now let $n_{t}(d y)$ denote the excursion entrance law for the process $\tilde{W}$ and denote by $n_{\lambda}(d x)$ its Laplace transform: $n_{\lambda}(d y)=\int_{\infty}^{1} e^{-\lambda t} n_{t}(d y) d t$. In particular, letting $\lambda \downarrow 0$ we get $n_{0}$, the Green function of the excursion law. The Laplace transform $n_{\lambda}$ of the excursion entrance law satisfies what is essentially the resolvent identity:

$$
n_{\varepsilon} \tilde{R}_{\lambda}^{\partial}=\frac{n_{\varepsilon}-n_{\lambda}}{\lambda-\varepsilon}
$$

for $\lambda \neq \varepsilon, \lambda, \varepsilon>0$. Furthermore, if $\tilde{W}$ does not spend positive time at $0, \tilde{R}_{\lambda} f(0)$ may be obtained from $n_{\lambda}$ using

$$
\tilde{R}_{\lambda} f(0)=\frac{n_{\lambda} f}{\lambda n_{\lambda} 1},
$$

where for any measure $\mu$ and any function $f$ we write $\mu f$ for $\int f d \mu$.

The key observation which enables us to explain the result (2.7) in terms of excursion theory is that $m_{1}$, which is an invariant measure for $\tilde{W}$, is a multiple of $n_{0}$, and without loss of generality we may assume that $m_{1}=n_{0}$. To see why this is the case, we now show that $n_{0}$ is also an invariant measure. For a fixed $\varepsilon>0$,

$$
\begin{aligned}
\lambda n_{\varepsilon} \tilde{R}_{\lambda} f & =\lambda n_{\varepsilon}\left(\tilde{R}_{\lambda}^{\partial} f+\psi_{\lambda} \tilde{R}_{\lambda} f(0)\right) \\
& =\lambda \frac{n_{\varepsilon}-n_{\lambda}}{\lambda-\varepsilon} f+\lambda n_{\varepsilon}\left(1-\left(\lambda \tilde{R}_{\lambda}^{\partial} 1\right)\right) \tilde{R}_{\lambda} f(0) \\
& =\frac{\lambda}{\lambda-\varepsilon}\left(n_{\varepsilon}-n_{\lambda}\right) f+\lambda\left(n_{\varepsilon} 1-\frac{\lambda}{\lambda-\varepsilon}\left(n_{\varepsilon}-n_{\lambda}\right) 1\right) \tilde{R}_{\lambda} f(0) \\
& =\frac{\lambda}{\lambda-\varepsilon}\left(n_{\varepsilon}-n_{\lambda}\right) f+\lambda\left(\frac{\lambda}{\lambda-\varepsilon} n_{\lambda} 1-\frac{\varepsilon n_{\varepsilon} 1}{\lambda-\varepsilon}\right) \tilde{R}_{\lambda} f(0) \\
& \rightarrow n_{0} f-n_{\lambda} f+\left(\lambda n_{\lambda} 1\right) \tilde{R}_{\lambda} f(0) \text { as } \varepsilon \downarrow 0 \\
& =n_{0} f, \text { as required. }
\end{aligned}
$$

(In the above we have used the fact that the lifetime $H$ of excursions is almost surely finite, which implies that $\varepsilon n_{\varepsilon} 1=\int\left(1-e^{-\varepsilon H(\omega)}\right) n(d \omega) \rightarrow 0$ as $\varepsilon \downarrow 0$, where $n$ is the Itô excursion measure and $n(H(\omega)=\infty)=0$.)

If $\tilde{W}$ does spend positive time at 0 , we have the following expression for $\tilde{R}_{\lambda} f(0)$ :

$$
\tilde{R}_{\lambda} f(0)=\frac{n_{\lambda} f+\gamma f(0)}{\lambda n_{\lambda} 1+\gamma \lambda},
$$

for some positive constant $\gamma$ (which we could usually take to be 1). A similar treatment then shows that $n_{0}+\gamma \delta_{0}$ is an invariant measure of $\tilde{W}$.

Since $m_{1}=n_{0}$, the quantity inside the exponent on the right-hand side of (2.7) may be expressed as

$$
\lambda x^{2} \int_{0}^{1}\left(1-\lambda \tilde{R}_{\lambda}^{\partial} 1(s)\right) m_{1}(d s)=\lambda x^{2}\left(n_{0} 1-\lambda \frac{n_{0} 1-n_{\lambda} 1}{\lambda}\right)=x^{2} \lambda n_{\lambda} 1 .
$$


Finally, we may interpret the quantity $\lambda n_{\lambda} 1$ in the following way. Suppose that we mark the excursions from 0 of our process $\tilde{W}$ at rate $\lambda$ : that is if $\tilde{\tau}$ is the time until the first mark (or the time between marks), then $\mathbb{P}(\tilde{\tau}>t)=e^{-\lambda t}$. The significance of $\lambda n_{\lambda} 1$ is that it is the local time rate of $\lambda$-marked excursions from 0 for the process $\tilde{W}$. In most examples we shall come across later, $\tilde{W}$ is actually a time-change of a Brownian motion $W$ in $(0,1]$. It is therefore easier if we could do calculations in terms of excursions of Brownian motion. Note that we time-change $W$ via the right-continuous inverse of the additive functional

$$
A_{t}=\int_{0}^{1} l_{t}^{y} m_{1}(d y)
$$

where $l_{t}^{y}, y \in[0,1]$ is the local time process of $W$, to obtain $\tilde{W}$. Hence marking the excursions of $\tilde{W}$ at rate $\lambda$ is equivalent to marking those of $W$ according to the measure $\lambda m_{1}$, or equivalently according to the additive functional $\lambda A_{t}$ : in other words, if $\tau$ is the time at which the first mark occurs then $\mathbb{P}(\tau>t \mid W)=e^{-\lambda A_{t}}$. Equivalently we could say that, conditional on $W$, the marks arrive as a timeinhomogeneous Poisson process where, conditional on $W$, the number of marks in the interval $[0, t]$ has a Poisson distribution with mean $\lambda A_{t}$. (In the case where $m_{1}(d t)=d t$ and $X$ is Brownian motion, so that $W^{\partial}$ is Brownian motion reflected at 1 and killed at 0 and $D=I, Q=\Gamma=I$, we just have marking at exponential rate $\lambda$.) Thus we could (and usually do) interpret the quantity $\lambda n_{\lambda} 1$ equivalently as the local time rate of $\lambda m_{1}$-marked excursions from 0 for the process $W$. Also, from the Ray-Knight argument used to obtain (2.5) when $X$ is a Brownian motion, we see that $\phi^{2}$ at (2.5) also admits the following interpretation:

$$
\begin{aligned}
\phi^{2} & =\mathbb{P}^{1}\left(W \text { reaches } 0 \text { without being } \lambda m_{1} \text {-marked }\right) \\
& =\mathbb{P}^{1}(\tilde{W} \text { reaches } 0 \text { without being } \lambda \text {-marked }) .
\end{aligned}
$$

(Here, $W$ is the Brownian motion on $(0,1]$ started at 1 and reflected at 1 , referred to in the Ray-Knight Theorem 2.2. The fact that the process $W$ in the Ray-Knight theorem happens to be the same as the Markov process $W^{\partial}$ obtained from the Green function $v(s, t)$ of $X$ when $X$ is Brownian motion is purely coincidental; as we have seen, this is not true if $X$ is a Brownian bridge.) We may therefore summarize these results by reinterpreting (2.7) (at least when $X$ is a Brownian motion) as

$$
\mathbb{E}^{0}\left[\exp \left\{-\lambda \int_{0}^{1}\left(X_{s}+x\right)^{2} m_{1}(d s)\right\}\right]=p^{1 / 2} e^{-d x^{2}},
$$

where $p=\mathbb{P}^{1}\left(W^{\partial}\right.$ reaches 0 without being $\lambda m_{1}$-marked $)$ and $d$ is the local time rate of $\lambda m_{1}$-marked excursions from 0 for the process $W$. The case where $X$ is a Brownian bridge can be interpreted similarly, once we have identified what the corresponding $p$ is using a Ray-Knight type argument. The main advantage of the result (2.7a) lies in its simplicity; as we shall see in the next section, the excursion ideas not only allow us to do calculations in cases where it may be difficult to find the resolvent density $\tilde{r}_{\lambda}^{\partial}$ by other means, they also enable us to obtain some very general but simple results for quite complicated polymer structures.

As has already been pointed out in the introduction, in the applications to polymer conformations, we really need to find the laws of quadratic functionals of 
the form

$$
\int_{0}^{1} X_{s}^{2} d \mu-\rho\left(\int_{0}^{1} X_{s} d \mu\right)^{2}
$$

for $0<\rho \leqq 1$. In particular, we wish to evaluate the partition function for the quadratic functional $(2.8)$ with $\rho^{-1}=\mu[0,1]$, so that $(2.8)$ is translation invariant. We can calculate the Laplace transform of functionals of the form (2.8) using the techniques we have developed in this section. The trick is to mix the parameter $\rho$ over a standard Gaussian distribution. Let $G$ be a standard $N(0,1)$ random variable. The key observation is that

$$
\mathbb{E}\left[\exp \left\{-\lambda\left(\int_{0}^{1} X_{s}^{2} d \mu-\rho\left(\int_{0}^{1} X_{s} d \mu\right)^{2}\right)\right\}\right]
$$

can be expressed as

$$
\mathbb{E}\left[\exp \left\{-\lambda \int_{0}^{1} X_{s}^{2} d \mu-G \sqrt{2 \lambda \rho} \int_{0}^{1} X_{s} d \mu\right\}\right] .
$$

Conditional on $G$, the expectation in (2.10) has the same form as the Laplace transforms considered earlier; therefore (2.10) can be calculated by first conditioning on $G$ and using the methods developed earlier to evaluate the conditional expectation, and then taking expectation with respect to $G$.

\section{Examples and Applications}

Example (i). We consider first the simplest possible case: that of a chain polymer modelled by the path of a Brownian motion $\left\{B_{t}: t \in[0,1]\right\}$. We are interested in the moment of inertia (2.8) of this polymer, where in (2.8) $X=B$ and $\mu$ is Lebesgue measure. In order to obtain the Laplace transform (2.9), we first condition on the $N(0,1)$ random variable $G$ and consider $(2.10)$. In this case $(2.10)$ has the same form as the left-hand side of (2.7), where $a \equiv G \sqrt{\rho /(2 \lambda)}$. As we have already seen in Sect. 2,

$$
\mathbb{E}^{0}\left[\exp \left\{-\lambda \int_{0}^{1} B_{s}^{2} d s\right\}\right]=(\cosh \theta)^{-1 / 2}
$$

where $\lambda=\theta^{2} / 2$ throughout this section. The $\lambda$-resolvent density (with respect to Lebesgue measure) is

$$
r_{\lambda}(s, t)=\frac{2 \sinh (\theta s) \cosh (\theta(1-t))}{\theta \cosh \theta}, \quad 0 \leqq s \leqq t \leqq 1
$$

(We drop the tilde and the $\partial$ for notational convenience.) For $s>t$ we define $r_{\lambda}$ using the fact that $r_{\lambda}(s, t)=r_{\lambda}(t, s)$. It is easy to check that

$$
\lambda \int_{0}^{1} \int_{0}^{1} r_{\lambda}(s, t) d s d t=1-\frac{\tanh \theta}{\theta}
$$


Hence (1.9) gives

$$
\begin{aligned}
& \mathbb{E}\left[\exp \left\{-\lambda \int_{0}^{1} B_{s}^{2} d s-\sqrt{2 \lambda \rho} G \int_{0}^{1} B_{s} d s\right\} \mid G\right] \\
& =(\cosh \theta)^{-1 / 2} \exp \left\{G^{2}\left(\frac{\rho}{2}-\frac{\rho \tanh \theta}{2 \theta}\right)\right\} .
\end{aligned}
$$

The right-hand side of (3.2) is simply the Laplace transform of a $\chi_{1}^{2}$ random variable, and so taking expectations in (3.2) gives

$$
\begin{aligned}
& \mathbb{E}^{0}\left[\exp \left\{-\lambda\left(\int_{0}^{1} B_{s}^{2} d s-\rho\left(\int_{0}^{1} B_{s} d s\right)^{2}\right)\right\}\right] \\
& =(\cosh \theta)^{-1 / 2}\left[1-2\left(\frac{\rho}{2}-\frac{\rho \tanh \theta}{2 \theta}\right)\right]^{-1 / 2} \\
& =\left[(1-\rho) \cosh \theta+\rho \frac{\sinh \theta}{\theta}\right]^{-1 / 2},
\end{aligned}
$$

which agrees with the answer given in Chan (1991) and Donati-Martin and Yor (1991) obtained using different methods. Also, note that our above calculations give

$$
\mathbb{E}^{0}\left[\exp \left\{-\lambda \int_{0}^{1}\left(B_{s}+x\right)^{2} d s\right\}\right]=(\cosh \theta)^{-1 / 2} \exp \left\{-x^{2} \frac{\theta}{2}-\tanh \theta\right\}
$$

comparing with $(2.7 \mathrm{a})$, we recognize

$$
\frac{\theta}{2} \tanh \theta
$$

as the local time rate of $\lambda$-marked excursions made by $W$ from 0 .

Example (ii). This technique easily generalizes to the case of a measure possessing a piecewise constant density. Consider the same example but this time in (2.8) let

$$
\mu(d t)= \begin{cases}\alpha^{2} d t & t \in[0, a] \\ \beta^{2} d t & t \in(a, 1]\end{cases}
$$

To spell things out a little, we have

$$
q(t)= \begin{cases}\alpha^{2} & t \in[0, a] \\ \beta^{2} & t \in(a, 1] \\ 0 & \text { otherwise }\end{cases}
$$

It is now a simple matter to compute the solution to the differential equation

$$
\frac{1}{2} \phi^{\prime \prime}=\lambda q \phi, \quad \phi(0)=1, \quad \phi^{\prime}(1-)=0
$$

with the matching condition that $\phi$ is $C^{1}$ at 1 . We obtain

$$
\phi(x)= \begin{cases}A\left[\cosh \theta \alpha(x-a)-\frac{\beta}{\alpha} \tanh \theta \beta b \sinh \theta \alpha(x-a)\right] & 0 \leqq x \leqq a, \\ A[\cosh \theta \beta(x-a)-\tanh \theta \beta b \sinh \theta \beta(x-a)] & a \leqq x \leqq 1\end{cases}
$$


where we have set $b=1-a$ and

$$
A=\left[\cosh \theta \alpha a+\frac{\beta}{\alpha} \tanh \theta \beta b \sinh \theta \alpha a\right]^{-1} .
$$

If we extend $\phi$ to the whole of $\mathbb{R}$ by setting $\phi(x)=\phi(1)$ for $x \geqq 1$ and $\phi(x)=\phi(0)=1$ for $x \leqq 0$, then the process

$$
M_{t}:=\phi\left(W_{t}^{+}\right) \exp \left\{-\lambda \int_{0}^{t} q\left(W_{s}\right) d s-\frac{1}{2} \phi^{\prime}(0) L_{t}\right\}
$$

is a martingale, where $L$ is the local time at 0 of the Brownian motion $W$. From this, we conclude using the optional sampling theorem that

$$
\begin{aligned}
\mathbb{P}^{1} & (W \text { reaches } 0 \text { without being } \lambda q \text {-marked }) \\
& \left.=\mathbb{E}^{1}\left\{-\lambda \int_{0}^{H_{0}} q\left(W_{s}\right) d s\right\}\right] \\
& =\phi(1) \\
& =\left[\cosh \theta \alpha a \cosh \theta \beta b+\frac{\beta}{\alpha} \sinh \theta \beta b \sinh \theta \alpha a\right]^{-1},
\end{aligned}
$$

and moreover that the local-time rate of $\lambda q$-marked excursions is

$$
-\frac{1}{2} \phi^{\prime}(0)=\frac{1}{2} A[\theta \alpha \sinh \theta \alpha a+\theta \beta \cosh \theta \alpha a \tanh \theta \beta b] .
$$

Accordingly Eq. (2.7a) takes the form

$$
\begin{aligned}
\mathbb{E}^{x}[\exp \{- & \left.\left.\lambda \int_{0}^{1} B_{s}^{2} d \mu\right\}\right]=\left(\cosh \theta \alpha a \cosh \theta \beta b+\frac{\beta}{\alpha} \sinh \theta \alpha a \sinh \theta \beta b\right)^{-1 / 2} \\
& \times \exp \left\{-x^{2}\left[\frac{\theta \alpha \sinh \theta \alpha a \cosh \theta \beta b+\theta \beta \cosh \theta \alpha a \sinh \theta \beta b}{2(\cosh \theta \alpha a \cosh \theta \beta b+(\beta / \alpha) \sinh \theta \alpha a \sinh \theta \beta b)}\right]\right\} .
\end{aligned}
$$

Mixing $x$ over the $N(0,1)$ distribution as before now gives

$$
\begin{aligned}
\mathbb{E}^{0}[\exp & \left.\left\{-\lambda\left(\int_{0}^{1} B_{s}^{2} d \mu-\rho\left(\int_{0}^{1} B_{s} d \mu\right)^{2}\right)\right\}\right] \\
= & {\left[\left(1-\rho\left(a \alpha^{2}+b \beta^{2}\right)\right)\left(\cosh \theta \beta b \cosh \theta \alpha a+\frac{\beta}{\alpha} \sinh \theta \beta b \sinh \theta \alpha a\right)\right.} \\
& \left.+\frac{\rho \alpha}{\theta}\left(\cosh \theta \beta b \sinh \theta \alpha a+\frac{\beta}{\alpha} \sinh \theta \beta b \cosh \theta \alpha a\right)\right]^{-1 / 2} .
\end{aligned}
$$

Example (iii). In this next example we let $X$ in (2.9) be a Brownian bridge of length 1 from 0 to 0 , and $\mu$ is again Lebesgue measure. The quadratic functional (2.8) 
corresponds to the moment of inertia of a ring polymer. The covariance function for $X$ in this case is $v(s, t)=s \wedge t-s t$, from which we get the Green's function $g(s, t)=2(s \wedge t-s t)$ for a Brownian motion on $(0,1)$ killed at 0 and 1 . The resolvent density (with respect to Lebesgue) for this process is

$$
r_{\lambda}(s, t)=\frac{2 \sinh (\theta s) \sinh (\theta(1-t))}{\theta \sinh \theta}, \quad 0 \leqq s \leqq t \leqq 1
$$

(For $s>t$ we again use $r_{\lambda}(s, t)=r_{\lambda}(t, s)$.) Hence

$$
\lambda \int_{0}^{1} \int_{0}^{1} r_{\lambda}(s, t) d s d t=1+\frac{2}{\theta}\left(\frac{1}{\sinh \theta}-\operatorname{coth} \theta\right) .
$$

We saw in Sect. 2 that

$$
\mathbb{E}^{0,0}\left[\exp \left\{-\lambda \int_{0}^{1} X_{s}^{2} d s\right\}\right]=\left(\frac{\sinh \theta}{\theta}\right)^{-1 / 2},
$$

and so, for $G \sim N(0,1),(2.7)$ gives

$$
\begin{aligned}
\mathbb{E}[\exp & \left.\left\{-\lambda \int_{0}^{1} X_{s}^{2} d s-\sqrt{2 \lambda \rho} G \int_{0}^{1} X_{s} d s\right\} \mid G\right] \\
= & \left(\frac{\sinh \theta}{\theta}\right)^{-1 / 2} \exp \left[G^{2}\left(\frac{\rho}{2}+\frac{\rho}{\theta}(\operatorname{cosech} \theta-\operatorname{coth} \theta)\right)\right] .
\end{aligned}
$$

(Recall that in this example also, $a \equiv G \sqrt{\rho / 2 \lambda}$.) Taking expectations in (3.9), we obtain

$$
\begin{aligned}
\mathbb{E}^{0,0}\left[\exp \left\{-\lambda\left(\int_{0}^{1} X_{s}^{2} d s-\rho\left(\int_{0}^{1} X_{s} d s\right)^{2}\right)\right\}\right] \\
=\left(\frac{\sinh \theta}{\theta}\right)^{-1 / 2}\left[1-\rho+\frac{2 \rho}{\theta}(\operatorname{coth} \theta-\operatorname{cosech} \theta)\right]^{-1 / 2} \\
=\left[(1-\rho) \frac{\sinh \theta}{\theta}+\frac{2 \rho}{\theta}\left(\frac{\cosh \theta-1}{\theta}\right)\right]^{-1 / 2} .
\end{aligned}
$$

Example (iv). From these simple examples, models for more complicated polymer shapes can be built up. One such example is a star-shaped polymer, which we model by $n$ independent Brownian motions of length 1 starting from 0 . For such a polymer we wish to calculate the partition function

$$
\phi=\mathbb{E}^{0}\left[\exp \left\{-\lambda\left(\int_{0}^{1} \sum_{i=1}^{n} B_{i}^{2}(s) d s-\frac{\rho}{n}\left(\int_{0}^{1} \sum_{i=1}^{n} B_{i}(s) d s\right)^{2}\right)\right\}\right] .
$$


Introducing $G \sim N(0,1)$ as before, we can calculate $\phi$ using the results (3.2) and (3.3) together with the independence of the $B_{i}$. Thus

$$
\begin{aligned}
\phi & =\mathbb{E}\left\{\mathbb{E}^{0}\left[\exp \left\{-\frac{\theta^{2}}{2} \int_{0}^{1} \sum_{i=1}^{n} B_{i}^{2}(s) d s-\theta G \sqrt{\frac{\rho}{n}} \int_{0}^{1} \sum_{i=1}^{n} B_{i}(s) d s\right\} \mid G\right]\right\} \\
& =\mathbb{E}\left\{\prod_{i=1}^{n} \mathbb{E}^{0}\left[\exp \left\{-\frac{\theta^{2}}{2} \int_{0}^{1} B_{i}^{2}(s) d s-\theta G \sqrt{\frac{\rho}{n}} \int_{0}^{1} B_{i}(s) d s\right\} \mid G\right]\right\} \\
& =\mathbb{E}\left\{\prod_{i=1}^{n} \exp \left[G^{2}\left(\frac{\rho}{2 n}-\frac{\rho \tanh \theta}{2 n \theta}\right)\right](\cosh \theta)^{-1 / 2}\right\} \\
& =(\cosh \theta)^{-n / 2}\left(1-\rho\left(1-\frac{\tanh \theta}{\theta}\right)\right)^{-1 / 2} .
\end{aligned}
$$

Example (v). Even more complicated polymers can be analysed using these methods. We now describe a general class of polymers of which the examples we have given so far are special cases. Consider a branching polymer structure which we model by a deterministically branching Brownian motion, $B$. This can be thought of as a Brownian motion indexed by a tree $\mathscr{T}$. The root of the tree, which we denote by 0 , is the time origin of the time graph of $B$. We suppose that $B_{0}=x$. Each (internal) node of the tree corresponds to a time when branching has occurred. A segment (branch) of the tree is defined to be the time interval between two nodes. The length of each segment is the length of time the corresponding path of the Brownian motion is run for. Let $\mu$ be a measure on the tree. The value of $\mu$ on any segment of the tree is to be interpreted as the resistance of the corresponding segment of the polymer in the flow. The partition function of the branching polymer structure is

$$
\phi=\mathbb{E}\left[\exp \left\{-\left(\int_{\mathscr{T}} B_{s}^{2} d \mu-\rho\left(\int_{\mathscr{T}} B_{s} d \mu\right)^{2}\right)\right\}\right],
$$

when we take $\rho=\mu(\mathscr{T})^{-1}$, making the expression (3.12) translation invariant. Define

$$
\hat{\phi}(v):=\mathbb{E}\left[\exp \left\{-\int_{\mathscr{T}} B_{s}^{2} d \mu+v \sqrt{2 \rho} \int_{\mathscr{T}} B_{s} d \mu\right\}\right] .
$$

Introducing $G \sim N(0,1)$ and mixing the parameter $v$ over $G$ as before gives $\mathbb{E}[\hat{\phi}(G)]=\phi$. Completing the square in (3.13) gives

$$
\hat{\phi}(v)=e^{v^{2} / 2} \mathbb{E}\left[\exp \left\{-\int_{\mathscr{T}}\left(B_{s}-v \sqrt{\rho / 2}\right)^{2} d \mu\right\}\right],
$$

so the key is to calculate

$$
\psi(x):=\mathbb{E}^{x}\left[\exp \left\{-\int_{\mathscr{T}} B_{s}^{2} d \mu\right\}\right],
$$

where $B_{0}=x$ is the starting value of the deterministically branching Brownian motion. 
Given a particular choice of node as the root of the tree $\mathscr{T}$ for a deterministically branching Brownian motion $B$, we can assign to each segment of $\mathscr{T}$ a particular direction in the following manner: consider a particle moving along a segment of the tree; define the direction on this segment to be the direction the particle would have to move in order to get closer to the root. Thus we can think of each segment as having an arrow pointing in this direction.

Given a deterministically branching Brownian motion $B$, we may associate to $B$ a Markov process with $\mathscr{T}$ as the state space in the manner described in Sect. 2. Of course, this Markov process (which we denoted by $W^{\partial}$ ) in this case is Brownian motion on $\mathscr{T}$ with reflection at the free ends and killed at the root; at any internal node, excursions are equally likely to go down any of the incident edges. We let $W$ denote the Brownian motion on $\mathscr{T}$ not killed at the root, but reflected there. For each choice of node $k$ as the root, and each segment $\sigma=(i, j)$ connecting nodes $i$ and $j$, where $i$ is closer to the chosen root than $j$, define

$$
P_{\sigma, k}:=\mathbb{P}^{j}\left(W^{\partial} \text { reaches } i \text { without being } \mu \text {-marked }\right)
$$

and

$$
\psi_{k}(x):=\mathbb{E}^{x}\left[\exp \left\{-\int_{\mathscr{T}} B_{s}^{2} d \mu\right\}\right],
$$

where node $k$ is chosen as the time origin of $B$ and $B_{k}=x$.

Theorem 3.1. Let $B$ be a deterministically branching Brownian motion indexed by tree $\mathscr{T}$ and choose node $k$ of $\mathscr{T}$ as the root. Then if $B_{k}=x$,

$$
\psi_{k}(x)=\left(\prod_{\sigma} P_{\sigma, k}\right)^{1 / 2} e^{-d_{k} x^{2}},
$$

where $d_{k}$ is the local time rate of $\mu$-marked excursions by $W$ from node $k$.

Proof. The proof is by induction on the number $n$ of nodes. The case $n=2$ was proved earlier at (2.7a). Now take $n>2$ and suppose that the theorem holds for any tree with at most $n-1$ nodes. Two cases must be considered.

Case (1). $k$ is an internal node. In this case, if there are $m>1$ edges incident at $k$, the tree $\mathscr{T}$ breaks into $m$ subtrees (with fewer than $n-1$ nodes) with $k$ as a common node. Given $B_{k}=x$, the behaviour of $B$ on each of these $m$ subtrees is independent, from which (3.15) follows.

Case (2). $k$ is an external node (i.e. free end). Let $\mathscr{T}^{\prime}$ denote the tree $\mathscr{T}$ with $k$ and its incident edge deleted, and let $j$ be the node in $\mathscr{T}^{\prime}$ joined to $k$. Let $J$ denote the edge $(k, j)$. Then

$$
\begin{aligned}
\psi_{k}(x) & :=\mathbb{E}^{x}\left[\exp \left\{-\int_{\mathscr{T}} B_{s}^{2} d \mu\right\}\right] \\
& =\mathbb{E}^{x}\left[\exp \left\{-\int_{J} B_{s}^{2} d \mu-\int_{\mathscr{T}^{\prime}} B_{s}^{2} d \mu\right\}\right] \\
& =\mathbb{E}^{x}\left[\exp \left\{-\int_{J} B_{s}^{2} d \mu-d_{j} B_{j}^{2}\right\}\right]\left(\prod_{\sigma \in \mathscr{T}^{\prime}} P_{\sigma, j}\right)^{1 / 2},
\end{aligned}
$$


using the induction hypothesis. Applying (2.7a) to the measure $m_{1}(d s)=\mu(d s)$ $+d_{j} \delta_{j}(d s)$ on $J$ yields

$$
\psi_{k}(x)=\exp \left(-d_{k} x^{2}\right)\left(P_{J, k} \prod_{\sigma \in \mathscr{T}^{\prime}} P_{\sigma, j}\right)^{1 / 2} .
$$

From (3.14), we have

$$
\hat{\phi}(v)=e^{v^{2} / 2} \mathbb{E}^{-v \sqrt{\rho / 2}}\left[\exp \left\{-\int_{\mathscr{T}} B_{s}^{2} d \mu\right\}\right],
$$

which, on mixing $v$ over the $N(0,1)$ distribution gives

$$
\begin{aligned}
\phi & =\left(\prod_{\sigma \in \mathscr{T}} P_{\sigma, n}\right)^{1 / 2} \mathbb{E}\left[\exp \left\{-G^{2}\left(\rho d_{n}-1\right) / 2\right\}\right] \\
& =\left(\frac{\Pi_{\mathscr{T}, n}}{\rho d_{n}}\right)^{1 / 2},
\end{aligned}
$$

where $\Pi_{\mathscr{T}, n}=\prod_{\sigma \in \mathscr{T}} P_{\sigma, n}$.

Obviously, the expression (3.16) must not depend on the choice of root, which we now verify by obtaining a "coordinate-free" expression for (3.16). Because we are working with a tree, we only need to check that the calculation for two adjacent nodes yields the same answer. Take the segment between nodes 1 and 2, say, and consider the tree $\mathscr{T}$ as being two trees $\mathscr{T}_{1}$ and $\mathscr{T}_{2}$ rooted respectively at nodes 1 and 2 and connected by a segment between these nodes. The restrictions of $\mu$ to $\mathscr{T}_{1}$ and $\mathscr{T}_{2}$ will be denoted respectively by $\mu_{1}$ and $\mu_{2}$. Let $n^{1}$ and $n^{2}$ denote the excursion measure of excursions by $W$ from 1 and 2 respectively. Thus for example, in the notation established by Theorem 3.1, $d_{2}=n^{2}$ ( $\mu$-marked excursions from 2). Let $D_{1}=n^{1}\left(\mu_{1}\right.$-marked excursions from 1 into $\left.\mathscr{T}_{1}\right)$ and define $D_{2}$ similarly. Let the connecting segment have length $T_{c}$ and measure $\mu_{c}$ which is the restriction of $\mu$ on it. Taking node 2 to be the root one obtains

$$
\phi^{2}=\frac{\Pi_{\mathscr{T}_{1,1}} \Pi_{\mathscr{F}_{2,2}} \mathbb{P}^{1}\left(W^{\partial} \text { reaches } 2 \text { without being } \mu \text {-marked }\right)}{\rho d_{2}} .
$$

Now let

$$
\begin{aligned}
b_{c} & =n^{2} \text { (excursions from } 2 \text { which reach } 1 \text { without being } \mu_{c} \text {-marked) } \\
& =n^{1} \text { (excursions from } 1 \text { which reach } 2 \text { without being } \mu_{c} \text {-marked) }
\end{aligned}
$$

and

$$
\begin{aligned}
& \left.a_{c}=n^{1} \text { (excursions from } 1 \text { which reach } 2 \text { or get } \mu_{c} \text {-marked }\right), \\
& \tilde{a}_{c}=n^{2} \text { (excursions from } 2 \text { which either reach } 1 \text { or get } \mu_{c} \text {-marked) } .
\end{aligned}
$$

Then

$$
\mathbb{P}^{1}\left(W^{\partial} \text { reaches } 2 \text { without being } \mu \text {-marked }\right)=\frac{b_{c}}{a_{c}+D_{1}}
$$

and

$$
d_{2}=D_{2}+\tilde{a}_{c}-\frac{b_{c}^{2}}{a_{c}+D_{1}} .
$$


Hence

$$
\phi^{2}=\frac{\Pi_{\mathscr{T}_{1}, 1} \Pi_{\mathscr{T}_{2}, 2} b_{c}}{\rho\left\{\left(a_{c}+D_{1}\right)\left(\tilde{a}_{c}+D_{2}\right)-b_{c}^{2}\right\}},
$$

which is clearly symmetric in 1 and 2 .

Example (vi). So far we have only considered polymer structures modelled on trees. By making use of our previous results for Brownian bridges it is possible to handle polymers with rings. Consider a graph which consists of a loop (which we think of as the path of a Brownian bridge of length $\tau$ from $x$ to $x$ ) with $n$ trees $\mathscr{T}_{m}$, $m=1, \ldots, n$, attached by node $k_{m}$ to the loop at time $T_{m}$. In this case

$$
\begin{aligned}
\psi(x) & =\mathbb{E}^{x, x}\left[\exp \left\{-\int_{0}^{\tau} X_{s}^{2} d \mu-\sum_{m=1}^{n} d_{m} X_{T_{m}}^{2}\right\}\right]\left(\prod_{m=1}^{n} \Pi_{\mathscr{T}_{m}, k_{m}}\right)^{1 / 2} \\
& =\mathbb{E}^{x, x}\left[\exp \left\{-\int_{0}^{\tau} X_{s}^{2} d \mu^{*}\right\}\right]\left(\prod_{m=1}^{n} \Pi_{\mathscr{T}_{m}, k_{m}}\right)^{1 / 2},
\end{aligned}
$$

where $X$ is now a Brownian bridge of length $\tau$ from $x$ to $x$ and $\mu^{*}(d t)=$ $\mu(d t)+\sum_{m=1}^{n} d_{m} \delta_{T_{m}}(d t)$. We have seen already how to deal with each of the factors appearing on the right-hand side of (3.18).

Obviously, the examples we have presented so far, especially the single chain polymer and the star-shaped polymer, are very simple trees and we leave the reader to check that Theorem 3.1 gives the same answers as the ones obtained earlier in this section. We conclude by illustrating the use of (3.17) with two further examples.

Example (vii). The first is a relatively simple one involving two particles joined by a single strand of polymer, which we model by a Brownian path of length $T$. Thus we take the measure of resistance on the tree to be

$$
\mu(d t)=\frac{\alpha^{2}}{2} d t+R_{1} \delta_{0}(d t)+R_{2} \delta_{T}(d t) .
$$

Choosing 0 to be the root, we have

$$
\begin{aligned}
b & =n^{0}(\text { excursions from } 0 \text { which reach } T \text { without being } \mu \text {-marked }) \\
& =n^{T}(\text { excursions from } T \text { which reach } 0 \text { without being } \mu \text {-marked }) \\
& =\frac{\alpha}{2} \operatorname{cosech} \alpha T, \\
a & =n^{T}(\text { excursions from } T \text { which either reach } 0 \text { or get } \mu \text {-marked }) \\
& =\frac{\alpha}{2} \operatorname{coth} \alpha T+R_{2}, \\
\tilde{a} & =n^{0}(\text { excursions from } 0 \text { which either reach } T \text { or get } \mu \text {-marked }) \\
& =\frac{\alpha}{2} \operatorname{coth} \alpha T+R_{1} .
\end{aligned}
$$

(Some of these calculations may need a little explanation: the fact that $2 b=\alpha \operatorname{cosech} \alpha T$ is shown in $\S$ VI.56 of Rogers and Williams (1987) (the positive 
masses at either end do not affect these calculations); next, if $R_{1}=R_{2}=0$ we know from Example (i) in this section that excursions from $T$ have probability $(\cosh \alpha T)^{-1}$ of reaching 0 without a mark and hence if $R_{1}=R_{2}=0$, we deduce that $a=\alpha / 2 \operatorname{coth} \alpha T$. The case of positive $R_{1}$ and $R_{2}$ now follows easily.) Since $D_{0}=D_{T}=0$, we then have from (3.17),

$$
\phi=\rho^{-1 / 2}\left(\frac{b}{a \tilde{a}-b^{2}}\right)^{1 / 2} .
$$

Example (viii). Consider next the more complicated structure of a "bottle brush" polymer. This has a "backbone" consisting of $n+1$ nodes, $m$ joined to $m+1$ for each $m=0,1, \ldots, n-1$; at each node $k$, for $k=1,2, \ldots, n-1$, a tree $\mathscr{T}_{k}$ rooted at node $k$ is attached. Let the rate of marked excursions into $\mathscr{T}_{k}$ from node $k$ be $S_{k}$ and let $S_{n}=0$. We let node $n$ be the root of the tree thus constructed and we have a measure $\mu$ on the tree as before. Then

$$
\left.\left.\psi_{\mathscr{T}, n}(x)=\left\{\prod_{k=1}^{n-1} \Pi_{\mathscr{T}_{k}}\right\}^{1 / 2} \prod_{k=0}^{n-1} \mathbb{P}^{k} \text { (no } \mu \text {-mark before hitting } k+1\right)\right\}^{1 / 2} e^{-d_{n} x^{2}}
$$

where $d_{n}$ is the rate of $\mu$-marked excursions from the root $n$. Define recursively

$$
\begin{aligned}
& D_{0}=0 \\
& D_{k}=S_{k}+\tilde{a}_{k}-\frac{b_{k}^{2}}{D_{k-1}+a_{k}},
\end{aligned}
$$

where, if $\mu_{k}$ is the restriction of $\mu$ to the segment connecting node $k$ to node $k-1$ and $n^{k}$ is the excursion measure of excursions from $k$, then

$$
\begin{aligned}
\tilde{a}_{k} & =n^{k}\left(\text { excursions which either contain a } \mu_{k} \text {-mark or reach } k-1\right) \\
a_{k} & \left.=n^{k-1} \text { (excursions which either contain a } \mu_{k} \text {-mark or reach } k\right) \\
b_{k} & =n^{k} \text { (excursions which reach } k-1 \text { unmarked) } \\
& =n^{k-1} \text { (excursions which reach } k \text { unmarked) } .
\end{aligned}
$$

The meaning of $D_{k}$ is that it is the rate of marked excursions from node $k$ into that part of the tree which is farther away from the root $n$; in particular we have $D_{n}=d_{n}$ and

$$
\mathbb{P}^{k}(\text { excursion reaches } k+1 \text { unmarked })=\frac{b_{k+1}}{a_{k+1}+D_{k}} .
$$

Hence using the same argument as that used to arrive at (3.13), we have

$$
\phi^{2}=\frac{1}{\rho d_{n}} \prod_{k=1}^{n} \frac{\Pi_{\mathscr{T}_{k}} b_{k}}{a_{k}+D_{k-1}},
$$

if we put $\Pi_{\mathscr{T}_{n}}=1$ for convenience. The simplest form we can take for the $\mathscr{T}_{k}$ is just a single strand of polymer, that is, a single-segment graph. This then gives a fish-bone polymer structure. If the $k^{\text {th }}$ strand has resistance measure $\alpha_{k}^{2} / 2 \times$ Lebesgue and length $T_{k}$ then

$$
S_{k}=\frac{\alpha_{k}}{2} \tanh \alpha_{k} T_{k}
$$


and $\Pi_{\mathscr{T}_{k}}=\operatorname{sech} \alpha_{k} T_{k}$. If $\mu_{k}=\beta_{k}^{2} / 2 \times$ Lebesgue then

$$
\begin{aligned}
\tilde{a}_{k}=a_{k} & =\frac{\beta_{k}}{2} \operatorname{coth} \beta_{k} \Delta T_{k}, \\
b_{k} & =\frac{\beta_{k}}{2} \operatorname{cosech} \beta_{k} \Delta T_{k},
\end{aligned}
$$

where $\Delta T_{k}$ is the length of segment joining nodes $k$ and $k-1$.

Once we have managed to deal with this bottle brush structure, we can apply the same methods to even more complicated structures, for example, by attaching bottle brush polymers to each of the nodes $1,2, \ldots, n-1$. The possibilities are endless!

\section{References}

Chan, T: Indefinite quadratic functionals of Gaussian processes and least-action paths Ann. Inst H Poincaré 27, 2 (1991)

Chiang, T S., Chow, Y, Lee, Y J.: An abstract Wiener space approach to certain functional integrals. Preprint (1991)

Donati-Martin, C, Yor, M.: Fubini's theorem for double Wiener integrals and the variance of the Brownian path. Ann. Inst. H Poincaré 27, 2 (1991)

Duplantier, B : Areas of planar Brownian curves J. Phys. A 22, 3033-3048 (1989)

Dynkin, E.B : Markov processes and random fields Bull AMS 3, 975-999 (1980)

Dynkin, E.B : Markov processes, random fields and Dirichlet spaces. Phys. Reports 77, 239-247 (1981)

Fixman, M.: Radius of gyration of polymer chains. J Chem. Phys. 36, 306-318 (1962)

Gaveau, B : Principe de moindre action, propagation de chaleur et estimées sous-elliptiques sur certains groupes nilpotents Acta Math. 139, 95-153 (1977)

Helfer, A.D, Zhao, Z: Gaussian integration on Wiener spaces J Appl Prob 29, 46-55 (1992)

Krée, P.: A remark on Paul Lévy's stochastic area formula In: J Barroso (ed.) Aspects of Mathematics and its Applications. Elsevier (1986)

Lévy, P: Wiener's random function and other Laplacian random functions In: J Neyman (ed) Proceedings of the Second Berkeley Symposium in Mathematical Statistics and Probability 171-187 (1951)

McAonghusa, P., Pulé, J.V: An extension of Lévy's stochastic area formula Stochastics \& Stochastics Reports 26, 247-255 (1989)

McGill, P : A direct proof of the Ray-Knight Theorem. In: Séminaire de Probabilités XV, LMN 850, Berlin, Heidelberg, New York: Springer, pp 206-209 (1981)

Revuz, D, Yor, M : Continuous Martingales and Brownian Motion Berlin, Heidelberg, New York: Springer 1991

Rogers, L C.G : Itô excursion theory via resolvents. Z. Wahrscheinlichkeitstheorie verw. Gebiete 63, 237-255 (1983)

Rogers, L C G : A guided tour through excursions Bull. LMS 21, 305-341 (1985)

Rogers, L C G., Williams, D: Diffusions, Markov Processes and Martingales. Volume 2: Itô Calculus, Wiley (1987)

Rogers, L.C G., Shi, Z: Quadratic functionals of Gaussian processes, optimal control and the 'Colditz' example. Stochastics 41, 201-218 (1992)

Williams, D : Decomposing the Brownian path Bull Am. Math Soc. 76, 871-873 (1970)

Williams, D : Some basic theorems on harnesses In: D G Kendall, E F Harding (eds) Stochastic Analysis. New York: Wiley, pp 349-366 (1973)

Williams, D : Path decomposition and continuity of local time for one-dimensional diffusions, I Proc. LMS 28, 738-768 (1974)

Yor, M : On stochastic areas and averages of planar Brownian motion J Phys A 22, 3049-3057 (1989) 
